\title{
COMPUTATION OF ADHESIVE FORCES DUE TO VAN DER WAALS AND CAPILLARY EFFECTS ON REALISTIC ROUGH SURFACES
}

\author{
R. Ardito ${ }^{1}$, A. Corigliano ${ }^{1}$, A. Frangi ${ }^{1}$, L. Magagnin $^{2}$, F. Rizzini ${ }^{1}$ \\ ${ }^{1}$ Department of Structural Engineering, Politecnico di Milano (alberto.corigliano@polimi.it) \\ ${ }^{2}$ Department Chemistry, Materials and Chemical Engineering, Politecnico di Milano
}

\begin{abstract}
The goal of this research is to provide an effective tool for the computation of adhesion energy in the case of surfaces which are as alike as possible to real-life Micro-ElectroMechanical Systems (MEMS). The basic data is represented by a refined geometrical model of rough surfaces, whose properties have been measured by means of cutting-edge technologies, such as Atomic Force Microscopy. The measured stochastic parameters have been used in order to obtain artificial rough surfaces. An important issue has been represented by the effective modeling of attractive force. Among the various causes of adhesion, we have considered the van der Waals and capillary forces, which are prevalent in the typical applications of MEMS. The former interaction has been modeled via Lennard-Jones potential and proximity force approximation. Capillary effects have been studied in a more detailed way, in view of the lack (to the Authors' knowledge) of a definitive numerical techniques. The numerical results are well compared to experimental outcomes, even though some work is still in progress.
\end{abstract}

Keywords: Micromechanics, Finite Element Analyses, Adhesion, MEMS, Rough Surfaces.

\section{INTRODUCTION}

The reliability of Micro-Electro-Mechanical Systems (MEMS) is a topic of paramount importance for technological advances: proper design and fabrication of micro-devices must ensure the perfect functioning both in standard exercise conditions and in extreme situations (e.g. accidental drop, mechanical and electrical shock, harsh environment, etc.). Among the various dangerous situations, spontaneous adhesion can seriously jeopardize the reliability of micro-electro-mechanical systems. In view of the high surface-to-volume ratio of MEMS, the adhesive forces between parts in contact may exceed the elastic restoring force: in this case, the components remain stuck to each other and the micro-machine could be completely unusable.

The aforementioned scenario is often addressed to in the literature as "stiction failure", where the word "stiction" is a neologism coined from "static friction". A large part of the available literature on stiction is devoted to experimental investigations. In [1], the interferometric measurement of the detached length in cantilever beams has been correlated, 
through a fracture mechanics approach, to the adhesive surface energy. Bachmann et al. [2] approached the problem in a different way, by performing pull-in tests on silicon suspended disc. Recently, the Authors in [3] exploited the pull-out behavior for an indirect measurement of adhesive forces through optical and electrostatic data.

Many efforts have been also devoted to the computational prediction of adhesion. It has been shown in [4] that the problem of adhesive sphere could be solved in a genuine Finite Element (FE) environment, by modeling the elastic parts through conventional finite elements and performing a contact analysis. More recently [5], the Lennard-Jones interatomic potential has been used in FE analysis in order to obtain an innovative formulation of frictionless contact problem. The stochastic nature of the actual rough surface has been considered in many papers, most of which [6] are based on simplified models of elastic-plastic deformation. In [7] a simpler model has been adopted, in the sense that rigid-plastic behavior of asperities has been considered and that adhesive forces have been estimated on the basis of the average surface separation.

The goal of this research is to provide a predictive tool for the adhesion energy in the case of surfaces which are as alike as possible to real-life MEMS.

The proposed numerical method for predicting adhesion is founded on Finite Element (FE) analyses. The basic data is represented by a refined geometrical model of rough surfaces. To this purposes, the properties of realistic silicon surfaces have been measured by means of cutting-edge technologies, such as Atomic Force Microscopy. The measured stochastic parameters have been used in order to obtain artificial rough surfaces, generated by a digital filter technique.

An important issue has been represented by the effective modeling of attractive force. Among the various causes of adhesion, we have considered the van der Waals and capillary forces, which are prevalent in the typical applications of MEMS. The former interaction has been modeled via Lennard-Jones potential and proximity force approximation, which has been shown in the literature to provide good results for silicon surfaces. Capillary effects have been studied in a more detailed way, in view of the lack (to the Authors' knowledge) of a definitive numerical techniques. The creation of a fluid meniscus between two isolated asperities has been simulated by means of a FE procedure, which exploits the membrane model for the meniscus surface and a dynamic relaxation technique in order to obtain the final shape at equilibrium. The obtained results have been compared with different simplified models: some of them have been found in the literature, the other (more refined) ones have been proposed by the Authors. The simplified models have been extended in order to achieve the estimate of capillary force on the whole rough surfaces.

Finally, adhesion energy is obtained by traction-separation curves. The numerical results are well compared to experimental outcomes, even though some work is still in progress for a better tuning of the numerical model.

\section{MEASUREMENT OF ROUGH SURFACES}

The understanding and simulation of adhesive phenomena necessitate of a precise description of the surface topography. In fact, both the contact area and the magnitude of adhe- 
sive forces (which depends on the local distance between the surfaces) are strongly influenced by the surface roughness, which is commonly defined as the height variation of the surface compared to the reference plane. Given a sample of surface heights $z_{i}, i=1, \ldots, N$, the roughness is characterized by the following statistic parameters:

$$
\begin{gathered}
r . m . s .=\frac{1}{N} \sum_{i=1}^{N} z_{i} \quad \text { (root mean square). } \\
S K=\frac{1}{\sigma^{3} N} \sum_{i=1}^{N}\left(z_{i}-m\right)^{3} \text { (skewness). } \\
K=\frac{1}{\sigma^{4} N} \sum_{i=1}^{N}\left(z_{i}-m\right)^{4} \text { (kurtosis). }
\end{gathered}
$$

where $m$ and $\sigma$ represent average and standard deviation of the sample, respectively. A twodimensional rough surface can be interpreted as an ergodic stochastic process, governed by the spatial auto-covariance function (ACF). The ACF of a casual function can be interpreted as the measure of how much precisely nearby values of the function can be predicted on the basis of local observations. It has been observed that many technically interesting surfaces have an exponential ACF: the directional measures of how fast the effect of a local observation decays are named correlation lengths $\left(\lambda_{x}^{*}, \lambda_{y}^{*}\right)$.

In this research, the roughness has been measured by means of the Atomic Force Microscope (AFM), which allows for a nano-meter mapping of a sample surface giving the distribution of heights over the considered area. This distribution is analyzed statistically and the resulting parameters are used in order to characterize the surface roughness. An extensive series of measures has been performed in order to achieve meaningful statistical data for the poly-silicon surface. The optimal measure should be performed on a large surface with high definition, but this is not compatible with the controlling software of the AFM, so there is a compromise between the sample area and the definition. In the poly-silicon surface the roughness of many small samples is measured in order to collect significant statistical data.

To obtain meaningful statistical data 26 measures on a $4 \times 4 \mu \mathrm{m}^{2}$ area are performed with a definition of $1024 \times 1024$ points, so with steps $3.9 \mathrm{~nm}$ long in both directions. Figure 1 shows a typical surface topography with the poly-silicon grains. It is evident the effect of the measure direction: some vertical stripes can be noted on Figure 1.

Table 1 reports the statistical descriptors of roughness. The r.m.s. is much higher than the typical values for single-crystal silicon surface: this is due to the effect of grain structure. It is not possible to define the correlation length in the orthogonal direction to the measurements, due to the instrumental capabilities. However, it is reasonable to assume an isotropic surface, i.e. equal correlation lengths in both directions. The distribution seems to be Gaussian and this is confirmed by the values of skewness and kurtosis, reported in Table 1. In fact, such values can be considered practically equal to the Gaussian values ( $S K=0, K=3$ ) because their discrepancy w.r.t. the latter is lower than their standard deviation.

Table 1. Statistical descriptors of the poly-silicon surface roughness measured via AFM. 


\begin{tabular}{cccc}
\hline statistical descriptor & average & std & std \% \\
\hline r.m.s. & $9.3869[\mathrm{~nm}]$ & $\pm 2.0892[\mathrm{~nm}]$ & $\pm 22.2563 \%$ \\
SK & 0.0477 & \pm 0.28621 & $\pm 599.1 \%$ \\
$\mathrm{~K}$ & 3.6439 & \pm 0.65567 & $\pm 17.9936 \%$ \\
$\lambda_{x}^{*}$ & undefined & & \\
$\lambda_{y}^{*}$ & $298.22[\mathrm{~nm}]$ & $\pm 113.3[\mathrm{~nm}]$ & $\pm 37.99 \%$ \\
\hline
\end{tabular}

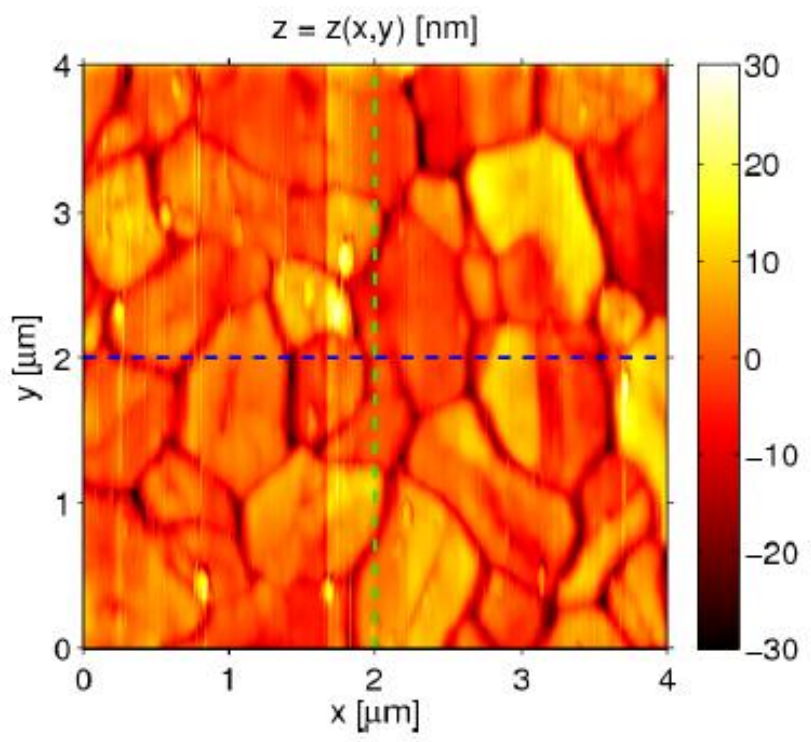

Figure 1. AFM topography measurements for the poly-silicon surface.

\section{MODELS FOR THE ADHESIVE FORCES}

\subsection{Van der Waals forces}

It is worthwhile to underline some essential aspect of the van der Waals forces: effectiveness from interatomic spacing (more or less $0.2 \mathrm{~nm}$ ) up to distance greater than $10 \mathrm{~nm}$; change of sign depending on the material properties (usually attractive force, but can change in repulsive); anisotropic behavior in some cases of interactions; "non-additivity", due to the fact that the dispersion force between two atoms is affected by the presence of a third atom.

Considering that in this work we focus on the overall force between two surfaces, this last observation is very important. The van der Waals force can be computed on the basis of the atom-to-atom potential, which depends the distance $\mathrm{D}$ between the atoms (or molecules) and is governed by the Hamaker constant, which has the following value for silicon:

$$
A=27 \cdot 10^{-20} J
$$

Lennard-Jones potential [8] is widely used. This potential (see Figure 2) captures the mild attraction $\left(6^{\text {th }}\right.$ power) of surfaces as they approach one another and the strong repulsion $\left(12^{\text {th }}\right.$ power), or steric forces, when they come too close to one another. 


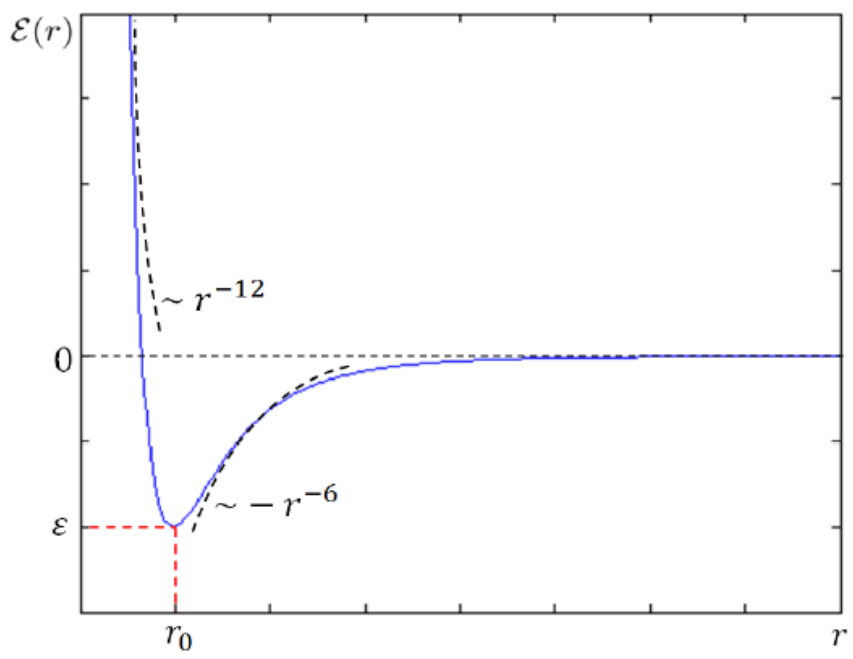

Figure 2. Schematic representation of the Lennard-Jones potential for van der Waals force.

The pressure consequent to van der Waals interactions is computed in the framework of the so-called "Proximity Force Approximation" (PFA) [9]. Each point on a surface is endowed with a portion of area and interacts with a corresponding point on the opposite surface. The van der Waals force is then computed by introducing the following pressure (based on Lennard-Jones potential) on the portion of surface connected to each point:

$$
q_{v d W}(r)=\frac{A}{6 \pi\left[D(r)+z_{0}\right]^{3}}\left[\left(\frac{z_{0}}{D(r)+z_{0}}\right)^{6}-1\right] .
$$

In the previous equation, $r$ is the lateral position on the surface and $z_{0}$ is the equilibrium separation, which for silicon reads:

$$
z_{0}=0.149 \mathrm{~nm}
$$

The overall force is obtained by a summation of the contributions provided by all the points.

\subsection{Capillary attraction}

The evaluation of capillary attraction is based on the concept of "surface tension", which is indicated by $\gamma_{L}$ and measured in units of force per unit length (e.g. N/m). The presence of surface tension in the fluid involves the possible creation of a fluid meniscus, depending on the "contact angle" $\theta$, which can be easily measured during experiments. If the contact angle is less than $90^{\circ}$, an attractive force arises between two wet plates. Moreover, fluids that show a small value of the contact angle (i.e. fluids that "wet" the solid substance) will spontaneously condense from vapor into bulk liquid: for this reason, capillary attraction arises in a humid environment.

At equilibrium, the meniscus curvature is related to the relative humidity $(\mathrm{RH})$ by the Kelvin equation: 


$$
r_{K}=\frac{\gamma_{L} \cdot V}{R \cdot T \cdot \log R H}
$$

where $V=18 \cdot 10^{-6} \mathrm{~m}^{3}$ is the molar volume of water, $R=8.3143 \mathrm{~J} / \mathrm{mol} / \mathrm{K}$ the gas constant and $\mathrm{T}$ the absolute temperature. The corresponding Laplace pressure reads $q_{L}=\gamma_{L} / r_{K}$.

The computation of the capillary forces is subordinated to the evaluation of the shape of the multiple menisci in different positions of the rough surface. The solution of such a problem represents a formidable task: to the Authors' knowledge, nobody has formulated a suitable algorithm for the case of surfaces with generic shape. A simple (though inexact) estimate can be obtained by considering an adaptation of the aforementioned PFA (see [7] and [8]). The force is computed point-wise by considering that the capillary attraction is kept constant and equal to the Laplace pressure until a critical separation is reached, after which the force is suddenly dropped to zero:

$$
\begin{array}{ll}
q_{c}=\gamma_{L} / r_{k} & D \leq 2 r_{K} \cos \theta \\
q_{c}=0 & D>2 r_{K} \cos \theta
\end{array} .
$$

The application of PFA, which is commonly accepted for van der Waals forces, can be criticized in the case of capillary attraction. For this reason, we have provided a set of alternative methods, referred to the simple case of two interacting spheres. First, we have considered an extension of the method proposed by [10], in order to compute the force on the basis of the exact geometry of the meniscus. Second, we have devised three approximate method (M1M3), based on the hypothesis that the meniscus assumes a spherical shape (Figure 3).

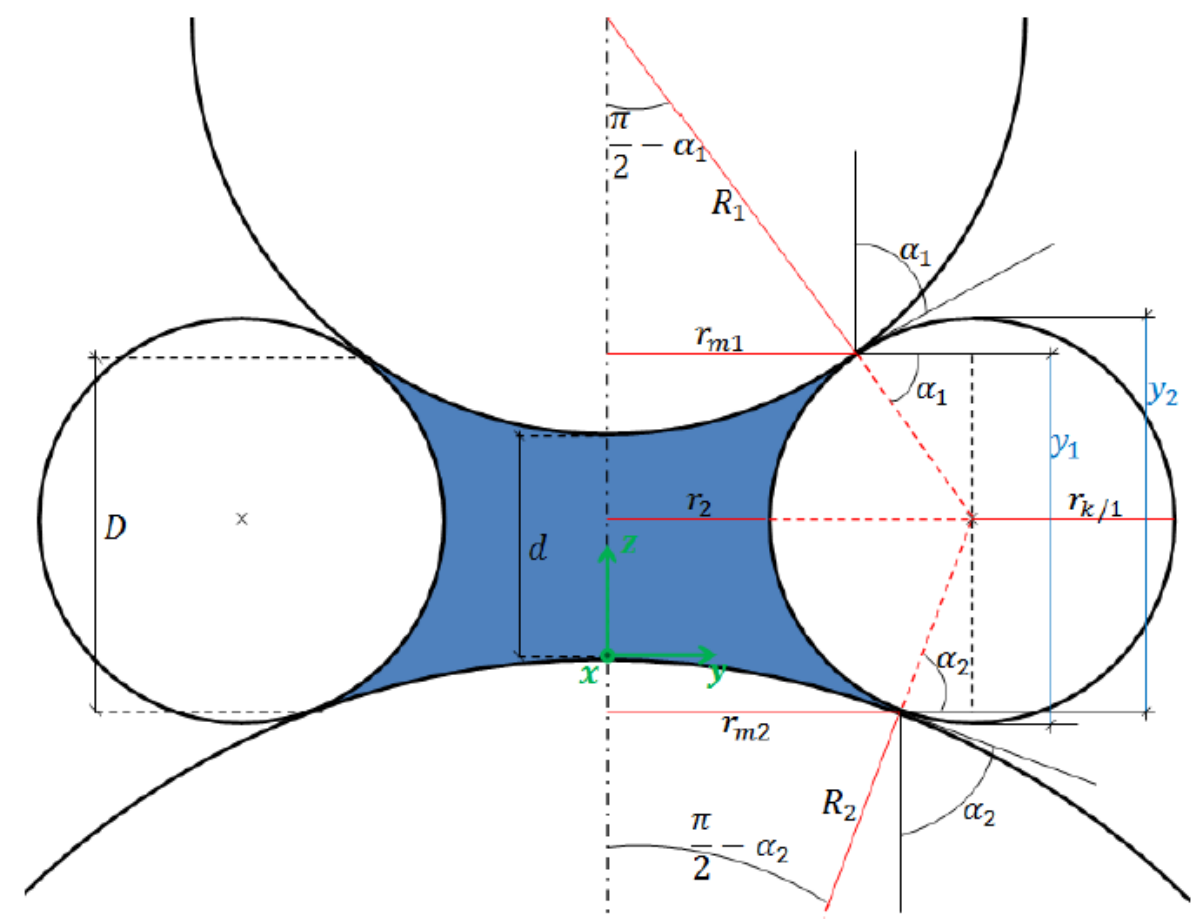

Figure 3. Geometric model for the approximate evaluation of capillary attractions between two spheres (models M1-M3). 
Third, we have computed the shape of the meniscus by means of the Finite Element Method, considering that the meniscus can be modeled as a structural membrane tensioned by the surface tension. Further details of the proposed methods will be presented in a forthcoming paper.

Figure 4 shows a comparative assessment of accuracy of the proposed methods, for the specific case of contact angle equal to zero (perfectly hydrophilic surfaces); the considered spheres have different radii, namely $R_{1}=35 \mathrm{~nm}$ and $\mathrm{R}_{2}=140 \mathrm{~nm}$; the relative humidity is 0.8 . It is possible to conclude that the simplified models M2 and M3 are in excellent agreement both with the "exact geometry" method and with the FEM outcomes. Similar computations have been carried out for different environmental conditions and for non-zero contact angle. From the whole set of analyses, it is possible to conclude that the model M3 represents a robust tool for estimating the force between two spheres.

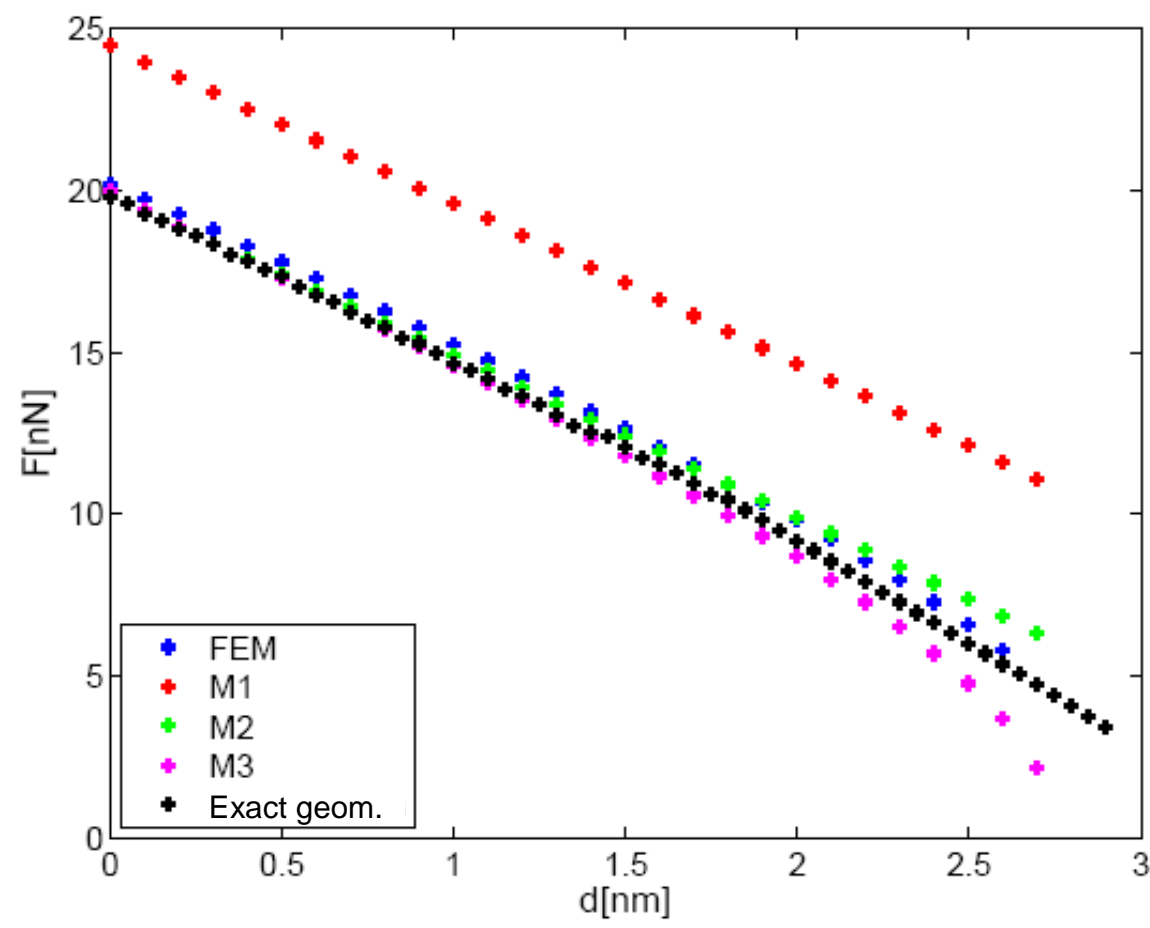

Figure 4. Capillary force vs. separation for the case of two spheres.

\section{GENERATION OF ARTIFICIAL ROUGH SURFACES}

The analyses have been based on a geometric model of the rough surface. Consequently, it has been necessary to resort to a specific algorithm which allows for the generation of artificial rough surfaces on the basis of the statistical descriptor.

As a first attempt, a 2D digital filtering technique has been adopted (Hu-Tonder method, [11]). The procedure can be summarized as follows. First, a sequence of random numbers $\eta(r, s)$ is generated, by extraction from a Gaussian distribution with zero mean and standard deviation equal to the given root mean square. The indices $(r, s)$ are used to identify a point in an evenly spaced grid. A Finite Impulse Response (FIR) filter is adopted in order to transform 
an input sequence of random numbers, $\eta(r, s)$, into an output sequence $z(r, s)$, which collects the heights of the rough surface. The coefficients of the filter are obtained by imposing that the output sequence is endowed with a specific ACF. That operation can be performed either by the application of direct convolution or by applying FFT techniques. The latter method appears to be the most effective one, both for the computing time and for the accuracy of the results. The plan view of the obtained surface is reported in Figure 5. In spite of the fact that the numerical method is unable to capture the grain structure (see Figure 1), it is worth noting that the distribution of the numerical and the measured heights almost coincide. Moreover, the ACF plots of the numerical and measured surfaces evidence a satisfactory agreement.

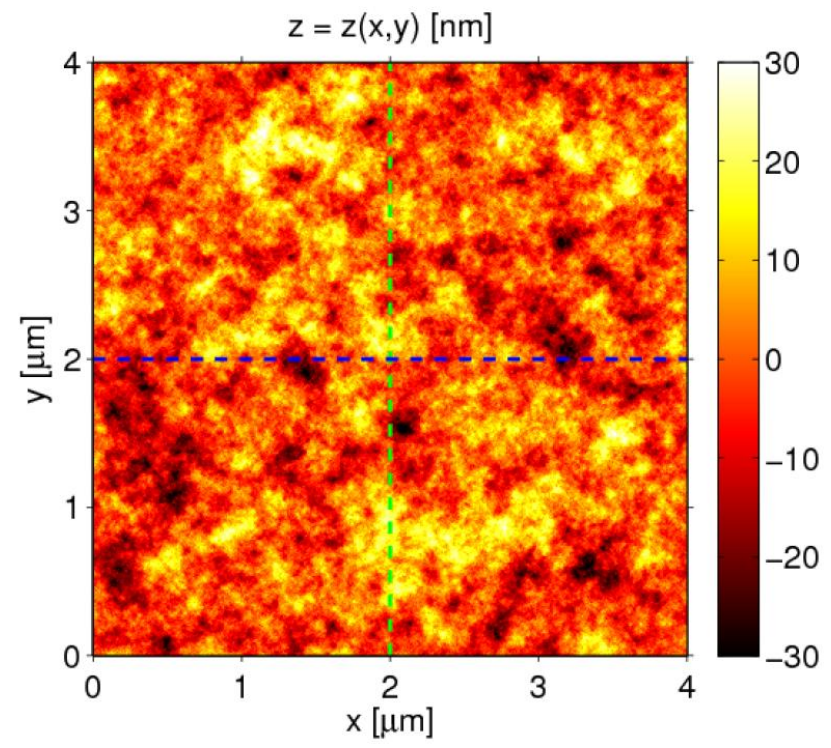

Figure 5. Topography of the numerically generated rough surface.

The artificial rough surface, generated in the aforementioned manner, is well-suited for the application of PFA for van der Waals and capillary forces. On the other hand, it is not possible to apply the new models M1-M3, which can be used only in the case of spherical asperities. Consequently, we have decide to implement a different method for obtaining rough surfaces, which are constituted by a set of spherical caps. First, the centers of the spheres are positioned on the basis of the measured correlation length. Then, the radii of the spheres are computed as the sum of a uniform value (bias) plus a random number extracted from a Gaussian distribution with zero mean and desired standard deviation. The bias is iteratively updated, in order to obtain a surface which is endowed with the correct r.m.s. and which is satisfactorily smooth. A typical output of the described procedure is reported in Figure 6. The numerical surface has been processed from the statistical point of view, obtaining good agreement with the measured distribution of heights and ACF. Moreover, from a qualitative point of view, the surface with spherical caps seems to be more representative of the granular structure of the considered surfaces. 


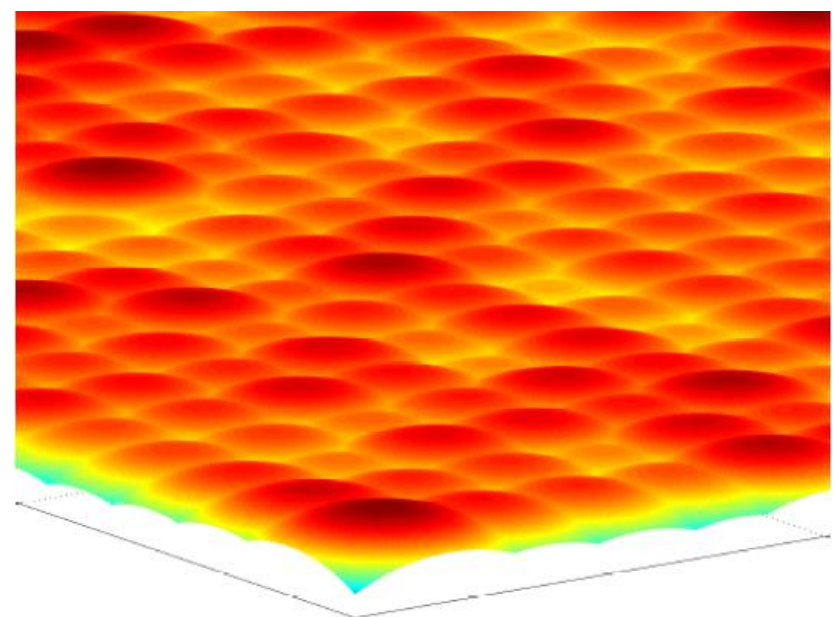

Figure 6. Detailed view of the numerical surface constituted by spherical caps. The same length scale is adopted both for in-plane and for out-of-plane dimensions.

\section{NUMERICAL RESULTS}

The numerical models for adhesive forces have been applied to the artificial rough surfaces described in Section 4. The surfaces obtained via the Hu-Tonder algorithm are suited only for the application of PFA, which has been considered both for the van der Waals and for the capillary force. Conversely, in the case of surfaces characterized by spherical caps, it is possible to adopt PFA and the semi-analytical methods. To this purpose, the models M1-M3 have been applied to each couple of spheres, located on the facing surfaces. The overall attractive force is obtained by simple summation of the contributions of all the couples of spheres. It is worth mentioning here that also the van der Waals interaction admits, for the case of spherical surfaces, the analytical solution. Its application is confined to the case of complete sphere, but it will be shown that the results are acceptable also for spherical caps.

The first analyses have been referred to the case of van der Waals force only, in order to compare the numerical outcomes to the experimental data reported in [9]. Figure 7 shows the adhesive energy for various roughness levels, ranging from $2.5 \mathrm{~nm}$ r.m.s. to more than 10 nm r.m.s. All the models catch the correct order of magnitude of adhesive energy and its trend with respect to roughness. Some expected differences arise between surfaces generated by the $\mathrm{Hu}$-Tonder algorithm and surface made by spherical caps. However, the examination of the results provides the confirmation of validity of PFA model for van der Waals forces and ensures that the representation by spherical caps does not alter the adhesive behavior of the surface.

Several analyses have been carried out for the case of capillary attraction, in the absence of van der Waals force. In particular, it has been considered that the opposite surfaces could be correlated, in the sense that the asperities show a specular pattern on the two surfaces, or uncorrelated. In view of the common features of real-life MEMS, only the latter case is considered herein. Figure 8 shows the specific adhesion force (over the nominal area of 25 $\mu \mathrm{m}^{2}$ ) for different roughness levels, ranging from $2 \mathrm{~nm}$ r.m.s to $10 \mathrm{~nm}$ r.m.s. Also the analytical solution for spherical bodies is reported. The results are obtained by assuming $\mathrm{RH}=0.8$. 


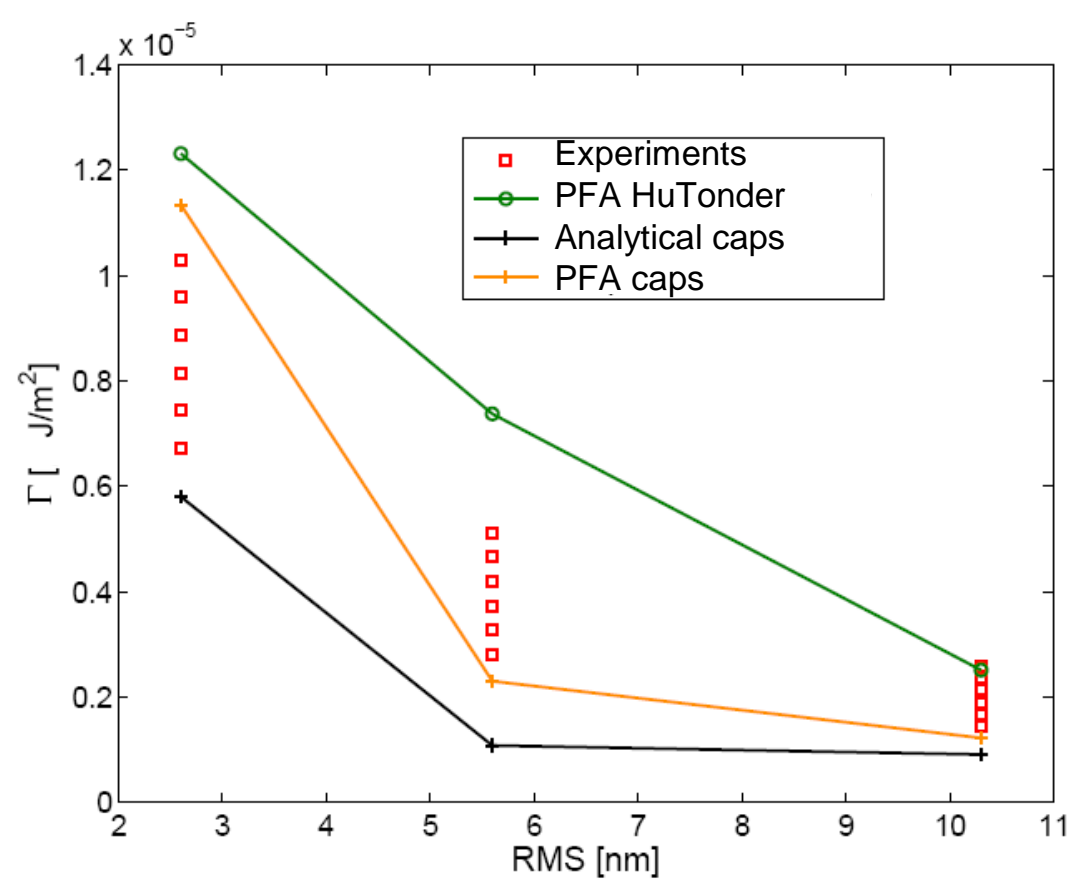

Figure 7. Adhesive energy vs. roughness r.m.s in the case of van der Waals forces only.

By examining Figure 8, it is possible to note that PFA provides similar results both for $\mathrm{Hu}$-Tonder surfaces and for spherical caps. The application of the more refined model M3 entails a slight reduction of force for roughness less than $4 \mathrm{~nm}$ r.m.s., while for higher roughness all the model seems to give similar estimates. Finally, it is possible to conclude that the analytical solution for two spheres provide strongly biased results.

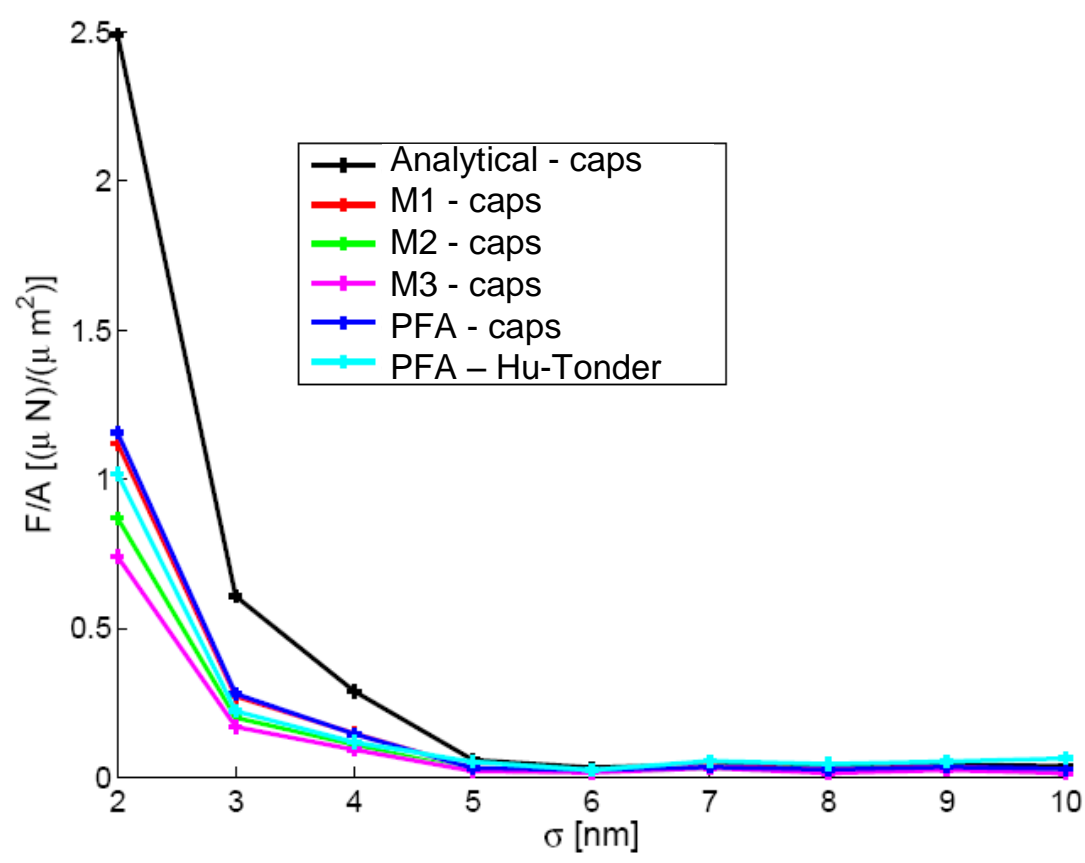

Figure 8. Specific adhesive force vs. roughness r.m.s in the case of capillary attraction only. Nominal area $25 \mu^{2} ; \mathrm{RH}=0.8$; zero contact angle. 
The previous analyses are executed by considering the minimum distance between the two surfaces, i.e. the distance which corresponds to contact of the bigger asperities. Figure 9 reports the results for different separations between the surfaces. Again, the analytical solution for spheres does not seem to provide reasonable results. Model M3 gives the smaller values, with similar trend with respect to PFA applied to Hu-Tonder surface.

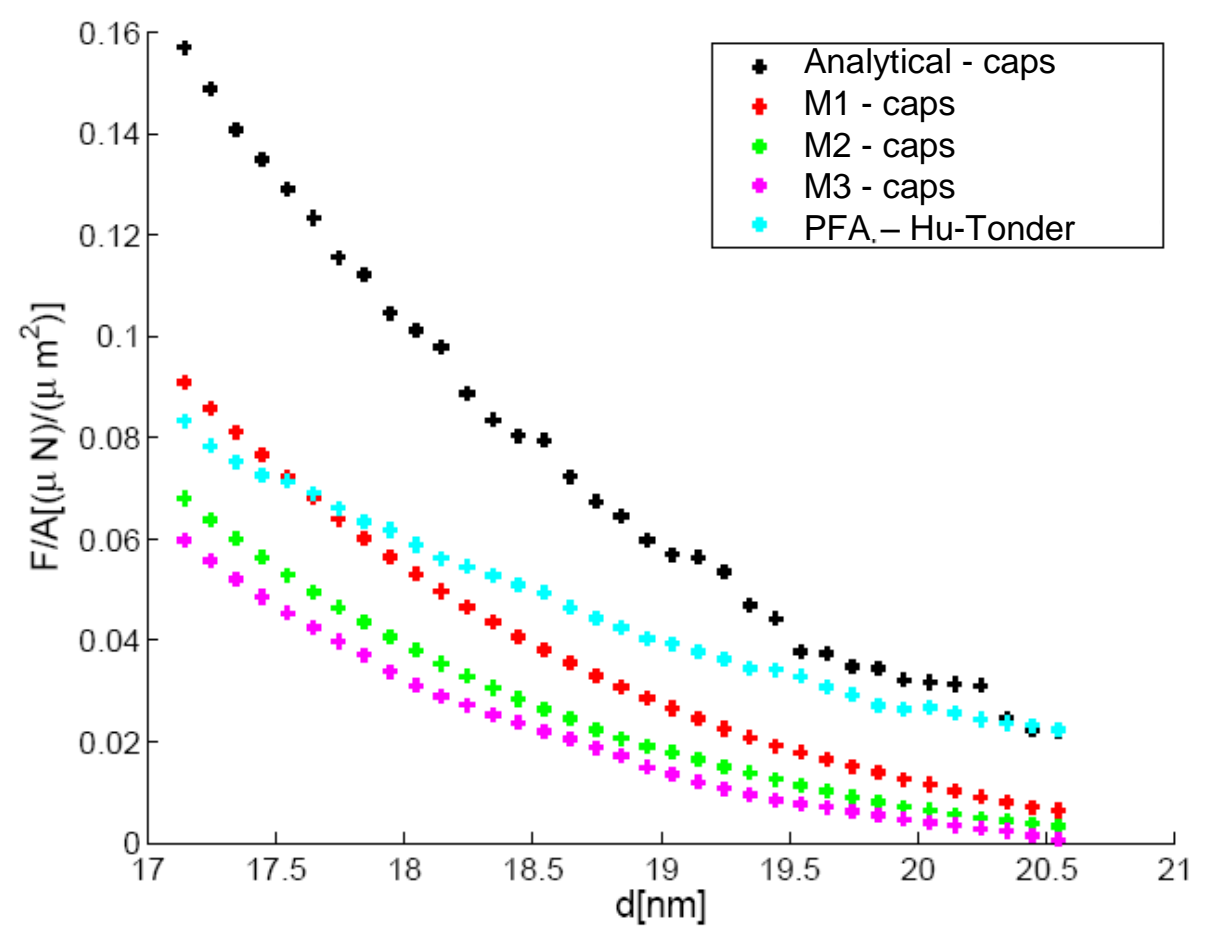

Figure 9. Specific adhesive force vs. separation distance in the case of capillary attraction only. Roughness $5 \mathrm{~nm}$ r.m.s.; nominal area $25 \mu^{2}$; $\mathrm{RH}=0.8$; zero contact angle.

\section{CONCLUSIONS}

The adhesion phenomena in MEMS has been studied from the computational point of view, with the purpose of obtaining a predictive tool for adhesion in different environmental situations. In particular, some improved models have been proposed for what regards the evaluation of capillary attraction. The results of the analyses lead to a positive reliability assessment of the models. In particular, the model for van der Waals force has been validated with respect to experimental data reported in the literature. A similar task is currently in execution for the case of capillary effect, but the model needs some improvement. In particular, it is necessary to include the effect of deformation of the asperities, which has not been considered so far. In fact, as a consequence of contact, the asperities may undergo elastic (recoverable) and plastic (permanent) deformations, with strong consequences on the overall adhesive behavior [12]. In the next future, the proposed semi-analytical model will be introduced in a Finite Element model of the representative surface, in order to obtain better estimates of adhesive energy. 


\section{Acknowledgements}

This work has been developed within the framework of the project funded by Cariplo foundation Cariplo09 "Surface interactions in micro and nano devices".

\section{REFERENCES}

[1] de Boer M.P., Michalske T.A., "Accurate method for determining adhesion of cantilever beams". J. Appl. Phys. 86, 817-827, 1999.

[2] Bachmann D., Kuhne S., Hierold C., "Determination of the adhesion energy of MEMS structures by applying Weibull-type distribution function”. Sens. Actuat. A 132, 407-414, 2006.

[3] Basu S., Prabhakar A., Bhattacharya E., "Estimation of stiction force from electrical and optical measurements on cantilever beams". J. MEMS 16, 629-634, 2007.

[4] Cho S-S., Park S., "Finite element modeling of adhesive contact using molecular potential". Trib. Internat. 37, 763-769, 2004.

[5] Sauer R.A., "A computational model for nanoscale adhesion between deformable solids and its application to gecko adhesion". J. Adhes. Sci. Techn. 24, 1807-1818, 2010.

[6] Hariri A., Zu J.W., Ben Mrad R., "Modeling of wet stiction in microelectro-mechanical systems (MEMS)". J. MEMS 16, 1276-1285, 2007.

[7] van Spengen W.M., Puers R., De Wolf I., "A physical model to predict stiction in MEMS”. J. Micromech. Microeng. 12, 702-713, 2002.

[8] Israelachvili J., Intermolecular and Surface Forces, Academic Press (London), 2011.

[9] DelRio F., de Boer M., Knapp J., Reedy E., Clews P., Dunn, M., "The role of van der Waals forces in adhesion of micromachined surfaces". Nature Mat. 4, 629-634, 2005.

[10] Pakarinen O.H., Foster A.S., Paajanen M., Kalinainen T., Katainen J., Makkonen I., Lahtinen J., Nieminen R. M., "Towards an accurate description of the capillary force in nanoparticle-surface interactions". Modell. and Simulat. Mat. Sci. and Eng. 13, 11751186, 2005.

[11] Hu Y.Z., Tonder K., "Simulation of 3D random surfaces by 2D digital filters and Fourier analysis". Int. J. Mach. Tools Manufact. 32, 83-90, 1992.

[12] Ardito R., Corigliano A., Frangi A., "Multiscale Finite Element models for predicting spontaneous adhesion in MEMS”. Mécanique \& Industries 11, 177-182, 2010. 